\title{
Chronische Encephalomyelitis disseminata oder multiple Sklerose?
}

\author{
Von \\ Hans Baumm.
}

(Aus der Sonderabteilung für Kriegsneurotiker am Festungshauptlazarett zu Königsberg i. Pr.)

(Eingegangen am 10. Juli 1919.)

Westphal hat im Jahre 1872 eine ,Affektion des Nervensystems nach Pocken und Typhus" beschrieben, die im wesentlichen durch akuten Beginn und die Symptome einer charakteristischen Sprachstörung, Veränderung des physiognomischen Ausdrucks, Ataxie, Zittern und psychische Störungen ausgezeichnet ist. Die Erscheinungen bildeten sich um einen gewissen Teil zurück, um dann auf einem geringeren Grade in ziemlich konstanter Weise monate- bis jahrelang unverändert fortzubestehen. Auf die Ähnlichkeit dieses Krankheitsbildes mit Charcots „Sclérose en plaques" weist Westphal hin. Leyden und andere Autoren haben später verwandte Krankheitsbilder als ,,akute Ataxie" beschrieben, eine Krankheit, die keinen Anspruch auf selbständige Stellung hat und daher nach dem Vorgange 0 ppenheims der akuten disseminierten Encephalomyelitis zugeteilt wird. Daß diese der multiplen Sklerose, namentlich ihrer akuten Form, sehr nahesteht, ja beide Erkrankungen ineinander übergehen können, ist bekannt.

Im folgenden seien 2 Fälle mitgeteilt, die mit den Westphalschen Kranken weitgehende Ähnlichkeit aufweisen, sich von diesen aber durch den von vornherein chronischen Verlauf unterscheiden.

Fall 1. Kriegsrentenempfänger E. B., ehemaliger Ulan, geb. 1885, Beruf Fischer.

Der Kranke gibt an, aus gesunder Familie zu stammen und selbst vor seiner Einstellung nie ernstlich krank gewesen zu sein, eine Aussage, die durch polizeiliche Vernehmung seiner Angehörigen sowie des Amtsvorstehers aktenmäßig bestätigt ist. Auf der Schule habe er mittelmäßig gelernt, später mäßig getrunken und geraucht. 1907/09 aktiv gedient bei einem Ulanenregiment, dann als „Dispositionsurlauber" entlassen. Verheiratet, 2 gesunde Kinder, ein ältestes im Alter von 6 Monaten gestorben. Er will nie geschlechtskrank gewesen sein.

Am 1. Mobilmachungstag eingezogen, kam er sofort ins Feld, wo er unter Erkältung infolge Übernachtens im Freien und schlechter Ernährung angeblich viel zu leiden hatte. Von November 1914 ab oft Magenschmerzen, Durchfälle und geschwollene Füße. 
Februar 1915 vorübergehend kurze Zeit wegen hornartiger Druckstellen an beiden Füßen in einem Reservelazarett, von wo er als dienstfähig zur Ersatzeskadron entlassen wurde. Nach wenigen Tagen leichten Dienstes wurde er wegen Schwielenbildung an beiden Füßen ins Festungshilfslazarett $X$. aufgenommen. Hier wurde im September 1915 festgestellt: Erhöhung aller Reflexe, erhebliche Abmagerung der Beine, Rombergsches Phänomen, Zittern der Hände und Pupillenträgheit. Diagnose: „Beginnende Tabes“. Wassermann im Vereinslazarett St.: negativ. - 22. XI. 1915. Verlegung nach Vereinslazarett T. zur spezialärztlichen Behandlung. Hier klagte B. über Beinschwäche, Schmerzen und Anschwellung der Fußgelenke, Kopfschmerzen, unruhigen Schlaf, schlechtes Gedächtnis und Stuhlträgheit. Objektiv bestand starke Schwielenbildung an den Füßen, geringere an den Handinnenflächen, unreiner erster Herzton, Entrun. dung und träge Reaktion der rechten Pupille, Flattern der Gesichts muskulatur, Zittern der Zunge und der Hände, Romberg, Sprachstörung, Atrophie der Beinmuskulatur, Gehstörung, Hyperhidrosis, auf geistigem Gebiet deutlicher Gedächtnis-und Merkdefekt, erschwerte Auffassung, dösiges Wesen und geringe elementare Kenntnisse. Im ganzen eine gewisse Geistesschwäche bei Euphorie. Öfters Durchfälle mit Schleim und Blut ohne Fieber, abwechselnd mit hartnäckiger Verstopfung. Nachts Knirschen mit den Zähnen und unruhiger Schlaf. Wassermann im Blut wieder negativ. Krankheitsbezeichnung: Organisches Gehirnleiden, mit großer Wahrscheinlichkeit beginnende progressive Paralyse. K. D. B. wurde angenommen. 30. IV. 1916 mit einer Rente von $50 \%$ entlassen.

Nachuntersuchung im Juli 1917 ergab: sehr geringes Fettpolster, dünne und schwache Muskulatur, stark ausgebildete Schwielen an Händen und Füßen, eingesunkenen Leib, Empfindlichkeit der Blinddarmgegend, träge Pupillen. reaktion, etwas belegte Zunge, schwankenden Gang, bei dem die Beine steif und unsicher aufgesetzt wurden, besonders starkes Schwanken bei ge. schlossenen A ugen, starren Blick, stockende und stotternde Sprache, Ataxie der Arme. Es wurde eine Verschlimmerung angenommen und die Rente auf $75 \%$ erhöht.

Nachuntersuchung im April $191^{\circ} 8$ stellte fest: Mäßigen Ernährungs- und Kräftezustand, steifen Gang, Fixation der Fußgelenke und der Zehen, diese in etwas gebeugter Stellung, etwas erhöh te Kniereflexe, geringes Lidflattern und Zittern bei Augen-und $F u B s c h l u B$, geringes Zittern der gespreizten Finger, Verdickung der Hohlhandschwielen an beidén Händen, stoßende Sprache. Die Krankheitsbezeichnung lautete diesmal „Kriegsneurose“, so daß B. nunmehr der Sonderabteilung für Kriegsneurotiker überwiesen wurde. Hier klagte B. über SchwindelgefühI und Schmerzen im ganzen Körper, besonders beim Gehen in den Hornhautstellen an den Fußsohlen. Er rege sich leicht auf, doch habe er das auch schon früher gehabt; daher habe er z. B. im ersten Jahre seiner Ehe mit seiner Frau oft Streit gehabt und dabei alles zusammengeschlagen, worüber er sich hinterher selbst geärgert habe. Jetzt bleibe ihm, wenn er sich a ufrege, gleich die Sprache fort und er habe ein Gefühl von Würgen in der Kehle. Früher sei er im allgemeinen ruhiger gewesen.

Es wurde folgender Befund erhoben: Größe $172 \mathrm{~cm}$, Brustumfang $80 / 86 \mathrm{~cm}$. Gewicht $56^{1} / 2 \mathrm{~kg}$, Temp. stets unter $37^{\circ}$, Puls regelmäßig, 60 i. d. Min. Beim Betasten des Bauches Gurren in der Blinddarmgegend, die etwas druckempfindlich war. Sonst keine Krankheitszeichen von seiten der inneren Organe, Urin frei von Eiweiß und Zucker. Derbe druckschmerzhafte Schwielenbildung an beiden Fußsohlen. In beiden Hohlhänden beginnende Du pu ytrensche Contractur.

Keine Klopfempfindlichkeit des Schädels, Pupillen gleich weit, rund, Reaktion auf Licht und Konvergenz beiderseits tadellos. Hornhaut o. B., insbesondere 
keine Pigmentierung. Augenbewegungen frei. Kein Nystagmus. Keine Besonderheiten am Augenhintergrund. Druckempfindlichkeit beider Supra- und Infraorbitalpunkte. Im übrigen keine krankhaften Störungen von seiten der Gehirnnerven. Leichtes Körperschwanken und Lidzittern beim Stehen mit geschlossenen Augen, lebhafte Dermographie, Zittern der mit gespreizten Fingern a usgestreckten Hände. Sehnenreflexe an beiden Körperhälften gleich und lebhaft. Keine Kloni. Kein Babinski. Bauchdeckenund Cremasterreflexe rechts wie links, von gewöhnlicher Stärke. Herabsetzung für alle Hautempfindungsqualitäten am linken Arm und der linken Rumpfhälfte. Die Sprache war verwaschen, stoßend, verlangsamt, ohne Modulation. Beim Sprechen wurde der Mund nur wenig geöfnet und die Gesichts- und Halsmuskulatur lebhaft mitinnerviert. Der Gesichtsausdruck war auch während des Sprechens merkwürdig starr. Der Gang war steif mit plantarwärts gekrümmten Zehen; nur der äußere Fußrand wurde aufgesetzt, wie der Kranke angab, um den schmerzhaften Druck auf die Fußsohlenschwielen zu vermeiden. Der Kniehackenversuch wurde beiderseits etwas unsicher ausgeführt, indessen ohne daß die Unsicherheit bei Augenschluß zunahm.

Die Wassermannsche Reaktion in Blut und Liquor war negativ. Der intradurale Druck war nicht erhöht. Der Liquor war wasserklar, enthielt im Kubikmillimeter 3 Zellen, zeigte aber eine deutlich positive Nonnesche Phase-I-Reaktion: Sofort auftretende Trübung nach Zusatz von AmmonSulfat-Lösung.

Psychisch war B. stets in jeder Richtung orientiert, geordnet und besonnen. Er machte einen gutmütigen, etwas e u phorischen Eindruck. Seine Schulkenntnisse waren sehr dürftig, er beherrschte nicht einmal das kleine Einmaleins. Die Merkfähigkeit war etwas herabgesetzt. In der Urteils- und Begriffsbildung entsprach er im wesentlichen seinem Stande und Bildungsgrade. Der Gesamteindruck war der eines einfältigen, von Hause aus wenig intelligenten Menschen, der sich um Dinge, die außerhalb seines beruflichen Gesichtskreises liegen, nie gekümmert hat.

Während seines 5 wöchigen Aufenthaltes auf der Abteilung blieb der Zustand im wesentlichen der gleiche. Eine kurze elektro-suggestive Behandlung (bevor der Kranke in meine Behandlung kam) brachte eine vorübergehende Zunahme der subjektiven Beschwerden. Nach Ausschneiden der schmerzhaften Hornhautstellen an den Fußsohlen und warmen Fußbädern wurde der Gang regelrecht. B. gab auf Befragen zu, auch schon vor seiner Einberufung zum Kriegsdienst von Zeit zu Zeit an schmerzhafter Schwielenbildung an den Füßen gelitten zu haben. Die oben genannte Störung der Hautsensibilität war später nicht mehr nachzuweisen. Im übrigen war der Befund bei der Entlassung der gleiche wie bei der Aufnahme. 23. XII. 1918 Entlassung als kr. u. mit 331/3\% Erwerbsbeschränkung.

Zusammenfassung: Ein früher nie ernstlich krank gewesener 29jähriger Mann, der bei Kriegsausbruch ins Feld kommt und hier seit November 1914 oft an Durchfällen und Magenschmerzen leidet, sucht im September 1915 wegen schmerzhafter Schwielenbildung an beiden Füßen, woran er auch schon vor der Kriegszeit gelitten hat, das Lazarett auf. Hier wird Erhöhung aller Reflexe, Abmagerung der Beine, Ro mberg sches Zeichen, Zittern der Hände und Pupillenträgheit festgestellt, „,beginnende Tabes" diagnostiziert und der Kranke auf eine Nervenstation verlegt. Daselbst ergibt die Untersuchung als weitere Krank- 
heitszeichen Flattern der Gesichtsmuskulatur, Entrundung der rechten Pupille, Hyperhidrosis, Gedächtnis- und Merkdefekt, erschwerte Auffassung, dösiges Wesen, geringe Kenntnisse, Euphorie, nachts Knirschen mit den Zähnen und unruhigen Schlaf. Öfters werden schleimig-blutige Durchfälle, die mit hartnäckiger Verstopfung abwechselten, beobachtet. Zweimalige Blutuntersuchung nach Wassermann ergibt negatives Resultat. Die Krankheitsbezeichnung lautet: „Organisches Gehirnleiden, mit großer Wahrscheinlichkeit beginnende progressive Paralyse." Darauf Einleitung des D. u.-Verfahrens und Entlassung mit $50 \%$ Rente. Nachuntersuchung im Juli 1917, bei der wieder träge Pupillenreaktion, starrer Blick, schwankender Gang, Ataxie der Arme, stockende und stotternde Sprache gefunden wird, hat wegen Verschlimmerung des Leidens Erhöhung der Rente auf $75 \%$ zur Folge. Im Gegensatz dazu stellt der Nachgutachter im April 1918 wegen erhöhter Kniereflexe, Lidflattern und Zittern beim Rombergschen Versuch, Zittern der Finger, stoßender Sprache und eigenartigen steifen Ganges die Diagnose „Kriegsneurose“.

Die darauf veranlaßte Beobachtung auf der Kriegsneurotikerabteilung ergibt folgendes: die sog. ,aktive" Behandlung führt zu keiner Besserung. Dagegen wird der Gang nach lokaler Behandlung der Fußschwielen (Hühneraugen) regelrecht. Die Gangstörung war keine psychogene, sondern die Krümmung der Zehen sowie das Auftreten auf den äußeren Fußrand bezweckten lediglich Entlastung der schmerzhaften Fußsohlenschwielen von dem Druck des Körpers. Dagegen sind als konstante Symptome, die bei der Entlassung wie bei der Aufnahme unverändert fortbestanden, $\mathrm{zu}$ nennen: Leichtes Rombergsches Zeichen, Händezittern, Lebhaftigkeit der Sehnenreflexe, lebhafte Dermographie, starrer Gesichtsausdruck, erschwerte und verlangsamte, stoßende, verwaschene, modulationslose Sprache, auf geistigem Gebiet geringe Intelligenz, gutmütige Euphorie. Vorübergehend wird eine Sensibilitätsstörung im Bereich der linken Körperhälfte festgestellt. Der Liquor weist als einziges Krankheitszeichen eine deutlich positive Phase-I-Reaktion auf.

Fall 2. G. S., Oberleutnant d. R., geb. 1879, Beruf Apotheker, ledig.

Der Kranke stammt angeblich aus gesunder Familie und will selbst vor dem Kriege stets gesund gewesen sein, rechtzeitig gehen und sprechen gelernt, nie an Bettnässen oder Anfällen irgendwelcher Art gelitten haben. Genitalinfektion und Alkoholmißbrauch werden geleugnet. 1906/07 Einj.-Freiw. bei einem Inf.-Reg. am 23. V. 1911 zum Leutnant d. R. befördert.

Am 1. Mobilmachungstag mit einem Inf. Reg. ins Feld gerückt, wurde er am 5. VIII. 1914 bei Kibarty durch Granatsplitter an beiden Oberschenkeln verwundet. Nach 24 stündigem Liegen auf freiem Feld geriet er in russische Gefangenschaft, wo er nach seiner Angabe in mehreren Lazaretten behandelt wurde. Im Lazarett Dünaburg will er im Herbst 1914 die $W$ und ro se durchgemacht haben. Danach habe sich allmählich eine Verlangsamung und Erschwe- 
rung der S prache eingestellt, eine Störung, die sich im Lauf der schlechten Behandlung während der Gefangenschaft verschlechtert habe. - Während wiederholter ärztlicher Untersuchungen hätten ihm österreichische Ärzte Zeichen gegeben, wie er sich verhalten müsse, um ausgetauscht zu werden. Er hätte einen schlürfenden Gang mit gebückter seniler Haltung markieren müssen. Der Kranke will dann beobachtet haben, wie die russischen Ärzte bei seinem Namen den Vermerk "Sclerosis multiplex" machten. Nach erfolgter Wundheilung Ende März 1915 aus dem Lazarett entlassen, habe er zunächst 4 Wochen in einem Gefängnis (als Vergeltungsmaßregel) und dann den Rest seiner Gefangenschaft in einem Lager in Westsibirien zugebracht. Die Sprachstörung habe unverändert fortbestarden.

Am 25. IIT. 1918 mit einem Krankentransport nach Deutschland zurückgekehrt, befand er sich von April bis Anfang Juli 1918 in der Militärkurstation Bad Oeynhausen, wodurch sich die Sprachstörung erheblich gebessert haben soll. Später will der Kranke während eines Urlaubs an einem Sprachkursus in Berlin teilgenommen haben, den er jedoch schon nach 6 Stunden wegen plötzlicher Zurückberufung zum Ersatzbataillon unterbrechen mußte. Hier habe er Innendienst, nur ausnahmsweise zur Vertretung Kompagniedienst getan. Anläßlich des D. u.-Verfahrens stellte der Truppenarzt im Entlassungszeugnis eine „,psychogene Sprachstörung" fest, die die Überweisung des Kranken auf die Sonderabteilung für Kriegsneurotiker zur Folge hatte.

Bei der Aufnahme klagte S. über Erschwerung der Sprache, Zittern der Hände, namentlich der linken, mit der er z. B. nicht essen könne, schlechten Schlaf, Durstgefühl, Ermüdbarkeit in beiden Beinen, namentlich dem linken. Er sei matt und schlapp und unfähig zur Aufnahme geregelter Berufsarbeit. Jedes körperliche Unbehagen, wie gelegentliche Verdauungsstörungen, machten ihn sofort äußerst schwach. Die Sprachstörung neh me bei Erregung zu.

Es wurde folgender Befund erhoben: Alter 39 Jahre, Größe $174 \mathrm{~cm}$. Brustumfang $93: 99 \mathrm{~cm}$, Gewicht $74,5 \mathrm{~kg}$, Temp. stets unter $37^{\circ}$, guter Ernährungsund Kräftezustand, etwas blasse Gesichtsfarbe, verbildete Ohrmuscheln. Keine Krankheitszeichen von seiten der inneren Organe. Trin frei von Eiweiß und Zucker.

Je eine reizlose, z. T. etwas eingezogene alte $\mathrm{Ha}$ utnarbe 1 . an der Innenseite des linken Oberschenkels in Handtellergröße, 2. auf seiner Beugeseite in Bohnengröße, 3. handbreit ober- und innerhalb davon von $6 \mathrm{~cm}$ Länge, 4 . an der Innenseite des rechten Oberschenkels in Fünfmarkstückgröße. Die Beweglichkeit in sämtlichen Gelenken beider Beine war frei und kraftvoll, der Gang regelrecht.

Keine Druck- und Klopfempfindlichkeit am Kopf. Pupillen rund, gleich und mittelweit, Reaktion auf Licht und Konvergenz tadellos. Hornhaut o. B. Augenbewegungen frei, kein Nystagmus. Augenhintergrund, insbesondere Sehnervenpapille ohne krankhafte Veränderung. Im Gesichtsa usdruck fiel eine eigenartige Starre auf. Der Mund wurde beim Sprechen und Lachen links mehr in nerviert als rechts, die Zunge gerade und ohne Zittern herausgestreckt, das Gaumensegel war gleichmäßig geformt. Die Sprache war langsam, erschwert, mit Anspannung der Halsmuskulatur, ohne Modulation, deutlich skandierend. Feinschlägiges Zittern der mit gespreizten Fingern ausgestreckten Hände, das namentlich an der linken Hand bei willkürlichen Bewegungen grobschlägiger wurde, keine Muskelatrophien, keine Ataxie, kein Rombergsches Zeichen. Lebhafte Dermographie. Die Hornhautreflexe und der Würgereflex waren von gewöhnlicher Stärke, der Ba uchdeckenreflex 
links oben schwach, links unten und rechts nicht sicher auslösbar, der Cremasterreflex beiderseits gleich, schwach, die Kniereflexe beiderseits gleich, lebhaft, die Achillesreflexe beiderseits von gewöhnlicher Stärke. Kein Patellar- oder Fußklonus. Babinski, Oppenheim, Mendel-Bechterew negativ. Oberflächen- und Tiefensensibilität sowie Lagesinn waren intakt. Keine Schriftstörung. Blasen- und Mastdarmfunktion regelrecht.

Bei der Lumbalpunktion floß tropfenweise wasserklarer Liquor ab. Der Liquor enthielt eine Zelle im Kubikmillimeter und wies eine de utlich positive Nonnesche Phase-I-Reaktion auf: Sofort auftretende Trübung nach Zusatz einer gleichen Menge Ammonsulfatlösung. Wassermann in Blut und Liquor negativ.

Psychisch war S. in jeder Richtung orientiert, geordnet und besonnen. Er liebte, wie er selbst angab, die Einsamkeit und machte während seines ganzen 9 wöchigen Aufenthaltes auf der Abteilung einen in jeder Beziehung soliden Eindruck, befolgte die ärztlichen Ratschläge mit großer Gewissenhaftigkeit. Der Aufnahme der Berufstätigkeit fühlte er sich nicht gewachsen. Sonst waren seelische Krankheitszeichen nicht nachweisbar.

S. macht auf der Abteilung eine erfolgreiche Abführkur gegen Oxyuren durch. Im übrigen bestand die Behandlung in Galvanisation und Bädern, ohne eine wesentliche Anderung zu erzielen. Bei der Entlassung war der Befund bis auf das Körpergewicht, das $71,5 \mathrm{~kg}$ betrug, der gleiche wie bei der Aufnahme. 14. III. 1919 Entlassung als dauernd garnisondienstunfähig.

Zusammenfassung: Ein stets gesund gewesener 35 jähriger Reserveoffizier gelangt im August 1914 mit größeren Weichteilverwundungen an beiden Oberschenkeln in russische Gefangenschaft, wo er einige Wochen später während der Lazarettbehandlung die Wundrose übersteht. Hierauf merkt er zum ersten Male eine im Laufe der schlechten Behandlung während der Gefangenschaft allmählich zunehmende Verlangsamung und Erschwerung der Sprache. Bei später folgenden Untersuchungen durch russische Ärzte markiert er schlürfenden Gang und senile Haltung und beobachtet dann, wie bei seinem Namen der Vermerk ,Sclerosis multiplex" gemacht wird. März 1918 aus der Gefangenschaft zurückgekehrt, besteht die Sprachstörung unverändert fort, bessert sich aber durch eine Kur in Oeynhausen. Bei dem nun folgenden D. u.-Verfahren stellt der Truppenarzt, „psychogene Sprachstörung“" fest. Daher Uberweisung auf die Kriegsneurotikerabteilung.

Als konstante Krankheitszeichen werden hier festgestellt: Starrer Gesichtsausdruck, Schwäche des rechten unteren Facialisastes, langsame, erschwerte, mit Anstrengung der Halsmuskulatur erfolgende, modulationslose, skandierende Sprache, feinschlägiges Zittern der Hände, das an der linken bei Willkürbewegung grobschlägiger wird, teilweises Fehlen der Bauchdecken-, Schwäche der Cremasterreflexe, Lebhaftigkeit der Kniereflexe, leichte Ermüdbarkeit sowohl im allgemeinen wie besonders in den Beinen, Zunahme der Sprachstörung bei Erregung. Der Liquor zeigt als einzige krankhafte Veränderung deutlich positive Phase-I-Reaktion. 
Die Ähnlichkeit der beiden Fälle untereinander ist deutlich. Das Gemeinsame sei noch einmal kurz zusammengefaßt: Jeder der beiden Kranken bietet jetzt Lebhaftigkeit der Sehnenreflexe, gesteigerte Dermographie, Händezittern, starren Gesichtsausdruck und eine modulationslose, verlangsamte, mit Anstrengung hervorgebrachte Sprache, die in dem einen Fall (1) verwaschen, in dem anderen (2) skandierend ist und in beiden bei Erregung schlechter wird. Bei beiden ist ferner der Wassermann in Blut und Liquor negativ, der Liquor außerdem frei von Zellen; dagegen zeigt er beidemal eine ausgesprochene Globulinvermehrung. In beiden Fällen fehlen jetzt Störungen von seiten der Augen. Außerdem konnte in Fall 1 leichtes Ro mberg sches Zeichen, Hyperhidrosis und vorübergehend eine Sensibilitätsstörung, in Fall 2 teilweises Fehlen der Bauchreflexe und geringe Differenz der unteren Facialisäste festgestellt werden. Der ausgesprochenen allgemeinen geistigen Beschränktheit im ersten Falle entspricht im zweiten nur ein gewisses persönliches Insuffizienzgefühl. Beide Male haben sich die Krankheitserscheinungen schleichend entwickelt, einmal (Fail 2) nachdem eine Wundrose vorangegangen war, im anderen Fall (Fall 1) nachdem öfters „Magenschmerzen und Durchfälle" aufgetreten waren. Hier konnten auch während des Lazarettaufenthaltes eine Zeitlang blutig-schleimige Durchfälle, abwechselnd mit Verstopfung festgestellt werden. Schließlich ist auch noch zu erwähnen, daß sich in unserm Fall 1 nach den früheren Krankengeschichten vorübergehende subakute Verschlimmerungen des Krankheitszustandes einstellten, in denen träge Pupillenreaktion, Entrundung einer Pupille, schwankender Gang, Ataxie der Arme und nachts Knirschen mit den Zähnen beobachtet wurden. Auf die in diesem Fall vorhandene Gangstörung, die, wie der Erfolg der Behandlung zeigt, durch die schmerzhafte Schwielenbildung an den Fußsohlen verursacht war, gehe ich nicht weiter ein, da sie mit den uns beschäftigenden nervösen Symptomen nicht zusammenhängt.

Mit den eingangs erwähnten Westphalschen Fällen haben unsere Kranken die besondere Art der Sprachstörung, die Veränderung des physiognomischen Ausdrucks, das Zittern gemeinsam, während Ataxie - diese nur vorübergehend - und deutliche psychische Veränderung nur in unserem Fall 1 festgestellt wurden. Außerdem fällt auch in unseren Fällen die Beziehung zu einer Infektionskrankheit auf: Fall 2 erkrankt im Anschluß an eine Wundrose, Fall 1 hat vor dem Ausbruch des Nervenleidens monatelang an Durchfällen und „Magenschmerzen“" und später an blutig-schleimigen Durchfällen gelitten, so daß der Gedanke an eine mehr subakute Ruhrerkrankung wohl nicht von der Hand zu weisen ist.

Wenn ich mich nunmehr der differentialdiagnostischen Besprechung zuwende, so glaube ich zunächst, eine luetische oder metaluetische 
Zentralerkrankung bei unseren beiden Kranken ausschließen zu können . Weder Blut - bei Fall 1 trotz mehrmaliger Untersuchung - noch Liquor zeigen die typische Komplementablenkung, der Liquor keine Zellvermehrung. Pupillenveränderungen, die zwar bei dem ersten Kranken vorübergehend gefunden wurden, bestehen jetzt nicht. Gürtelgefühl, lanzinierende Schmerzen, gastrische Krisen und andere tabische Symptome waren nicht nachweisbar. Die tatsächlich bestehende Geistesschwäche im ersten Fall darf nicht zu hoch bewertet werden, einmal weil ein Vergleich mit seinem Geisteszustand vor Kriegsbeginn nicht möglich ist, sodann weil feststeht, daß der Kranke nach seiner Entlassung vom Militär seinen Beruf als Fischer, wenn vielleicht auch nicht mehr in vollem Umfange, ausgeübt hat. Ferner läßt auch die Sprachstörung in beiden Fällen ein echtes Silbenstolpern vermissen. Tabes und Paralyse - beides wurde bei Fall 1 nacheinander festgestellt - sowie Hirnlues sind also mit Bestimmtheit auszuschließen. Die in Fall 1 vorübergehend festgestellte Anisokorie und Pupillenträgheit vermag an dieser Auffassung nichts zu ändern, da beide Symptome nach den Erfahrungen von E. Müller, Oppenheim u. a. auch bei multipler Sklerose und Encephalomyelitis keine ganz ungewöhnlichen Erscheinungen sind.

Schwieriger gestaltet sich schon die Frage der Unterscheidung zwischen disseminierter Encephalomyelitis und multipler Sklerose einerseits, diffuser Sklerose, Pseudosklerose und Wilsonscher Krankheit andererseits. Die Möglichkeit einer diffusen Sklerose, an die man nach D. Gerhardt stets denken muß, wenn in dem sonst gut ausgeprägten Bild der multiplen Sklerose Nystagmus fehlt und psychischer Verfall mehr und mehr zutage tritt, muß in Fall 1 erwogen werden. Indessen schreitet der geistige und körperliche Verfall bei der diffusen Sklerose fort, zu dem Skandieren der Sprache tritt Lallen und Näseln hinzu, zum Schluß besteht allgemeine Lähmung mit Dysphagie, so daß der Kranke dauernd ans Bett gefesselt ist, während längere Remissionen nicht dem Krankheitsbilde der diffusen Sklerose angehören; man sieht, ein wesentlich anderes Bild als es unser Fall l zeigt. Ferner spricht gegen Pseudosklerose mit Wilsonscher Krankheit, der gegenüber sich die Differentialdiagnose der multiplen Sklerose, wie 0 ppenheim $u$. a. gezeigt haben, äußerst schwierig gestalten kann, einmal, daß in unseren Fällen die Erkrankung nicht, wie typisch, im Entwicklungsalter, sondern erst mit 29 bzw. 35 Jahren eingesetzt hat; auch spielt bei unseren Kranken die Heredität keine Rolle, die dort so häufigen apoplektiformen und epileptiformen Anfälle fehlen, desgleichen vermissen wir eigentliche dysarthrische und dysphagische Störungen, den charakteristischen Pigmentsaum der Cornea und schließlich Leberund Milzsymptome. 
Daß es sich schließlich bei unseren Fällen nicht, wie beide Male, ehe die Kranken auf meine Abteilung gelangten, angenommen wurde, um ein funktionelles Leiden handelt, beweist die positive Phase-I-Reaktion, die, wie Nonne gelehrt hat, für die Differenzierung zwischen funktioneller und organischer Erkrankung des Nervensystems ausschlaggebend ist.

Nicht sicher zu unterscheiden ist meines Erachtens in unseren Fällen die Frage, ob multiple Sklerose oder Encephalomyelitis disseminata vorliegt. Auffallend ist das Fehlen von Nystagmus und Veränderungen am Augenhintergrund, während die Sprache nur bei Fall 2 typisch skandierend, bei Fall 1 mehr verwaschen ist. Das Zittern bei unseren Kranken ist außer bei Fall 2, wo es auf den linken Arm beschränkt ist, kein typisches Bewegungszittern, wie es für die multiple Sklerose pathognomonisch ist. Von den bekannten Frühsymptomen der multiplen Sklerose weist nur Fall 2 teilweises Fehlen der Abdominalreflexe auf. Dieser zeigt außerdem eine geringe Facialisdifferenz, wie sie auch Westphal bei zwei seiner Kranken beschreibt, ohne ihr besonderen pathologischen Wert zuzuschreiben, während Nonne sie zu den Frühsymptomen der multiplen Sklerose zu rechnen geneigt ist. Auch der positive Ausfall von Nonnes Phase-I-Reaktion spricht nicht direkt für multiple Sklerose. V. Reichmann fand sie stets negativ, Nonne unter 68 Fällen nur einmal stark positiv, während Siemerling und Raecke in $45 \%$ ihrer Fälle positiven Ausfall feststellten. Ich selbst habe bei meinem kleinen Material, seit ich auf diese Erscheinung achte, bei dem klassischen Charcotschen Symptomenbilde ebenfalls Globulinvermehrung im Liquor vermißt. Ich möchte daher vor der Hand gegen die Amahme einer multiplen Sklerose in unseren Fällen gewisse Zweifel hegen und zunächst annehmen, daß sich, einmal im Anschluß an eine Wundrose, ein andermal in Verbindung mit einer. ruhrartigen Darmerkrankung das Bild einer disseminierten Encephalomyelitis entwickelt hat. Derartige Fälle sind außer von Westphal auch von Gubler, P. Marie, Lenhartz, Finkelnburg, Schlesinger, Leyden, Ebstein u. a. mitgeteilt, doch handelt es sich stets um akute Erkrankungen. Bei unseren Kranken sahen wir einen von vornherein chronisch verlaufenden Krankheitsprozeß, dessen Hauptsymptom, die Sprachstörung, seit Jahren offenbar obne wesentliche Veränderung besteht. Oppenheim will eine chronische Myelitis bzw. Encephalitis als selbständige Erkrankung nicht gelten lassen, sondern sie nach klinischen Erscheinungen und anatomischem Befund der multiplen Sklerose zuteilen. E. Müller wiederum rechnet auf Grund seiner anatomischen Untersuchungen alle Fälle von akuter multipler Sklerose mit entzündlichen Veränderungen - die Globulinvermehrung im Liquor unserer Kranken deutet ebenfalls auf solche hin - zur sekundären Sklerose 
und meint, daß diese kaum jemals das vollentwickelte klassische Bild Charcots biete. Unsere Fälle, die Nystagmus, Veränderungen des Augenhintergrundes und auch ein typisches Bewegungszittern vermissen lassen, scheinen einstweilen diese Ansicht E. Müllers zu stützen, doch bleibt der weitere Verlauf abzuwarten. Ob wir bei dem gegenwärtigen Befunde klinisch die Diagnose multiple Sklerose, sekundäre Sklerose oder chronische Encephalomyelitis stellen, ist schließlich Geschmackssache. Ich begnüge mich hier, außer den bereits erwähnten Autoren auf die Arbeiten von Leube, Schüle, Jacob, Fraenkel, Marburg, Rönne und Wimmer, Anton und Wohlwill, A. Cramer, Hoffmann, Maas u. a. zu verweisen, aus denen die innige Verwandtschaft zwischen multipler Sklerose, Encephalitis und Myelitis hervorgeht; hat doch schon Charcot selbst die multiple Sklerose als nichts anderes als eine interstitielle, chronische, primitive Myelitis bzw. Encephalitis aufgefaßt.

Was mir an diesen beiden Fällen interessant und mitteilenswert erschien, ist einmal der von vornherein chronische Verlauf bei vorwiegend cerebralen Symptomen, die offenbare Beziehung zu einer gleichzeitig oder kurz vorher überstandenen akuten bzw. subakuten Infektionskrankheit, die charakteristische Sprachstörung, die das einzige konstante und mit Sicherheit organische Krankheitszeichen bildet, während Nystagmus, Augenhintergrundveränderungen und typisches Bewegungszittern fehlen, und schließlich die Globulinvermehrung in dem cyto- und serologisch normalen Liquor. Während beide Kranke unter der für einen alten Krieger wenig schmeichelhaften Krankheitsbezeichnung „Kriegsneurose“ der Sonderabteilung zugeführt wurden, ermöglichte die Lumbalpunktion sofort die Richtigstellung der Diagnose. Dieser bei richtiger Anwendung wohl stets harmlose Eingriff, sollte daher bei irgendwie unklaren Erkrankungen des Nervensystems nie unterlassen werden.

Während der Drucklegung hatte ich Gelegenheit, in der Ambulanz einen dritten derartigen Kranken zu sehen:

Der jetzt 49 jährige Landsturmmann A. K., von Beruf Tischler, hat 1892/94 aktiv gedient und wurde Februar 1915 eingezogen. Von August bis Dezember 1917 war er wegen $R \mathbf{u ~ h r}$ in Lazarettbehandlung. Es besteht jetzt neben einer Affektion der linken Lungenspitze undeutliche, aber keine näselnde oder skandierende Sprache, Schwäche des rechten unteren Facialis astes, Patellar-und Achillesareflexie, beiderseits gleich schwacher Abdominalreflex, eine Sensibilitätsstörung im Bereich von $L_{1}$ bis $L_{4}$, Neigung beim $R$ ombergschen Versuch nach hinten zu fallen. Der Blutdruck beträgt $127 \mathrm{~mm}$ RR. Der Wassermann war in Blut und Liquor negativ, der Liquor enthielt 10 Zellen im Kubikmillimeter und zeigte schwach positive Nonnesche Reaktion. 
Ich glaube auch hier eine chronische disseminierte Entzündung im Zentralnervensystem nach Ruhr annehmen zu müssen, die freilich in diesem Falle neben cerebralen auch ausgesprochene spinale Symptome aufweist.

\section{Literaturverzeichnis.}

1. Anton u. Wohlwill, Multiple nicht eitrige Encephalomyelitis und multiple Sklerose, Zeitschr. f. d. ges. Neur. u. Psych. 31. 1912.

2. Charcot, Leçons sur les maladies du système nerveux, T. I. 1880.

3. Cramer, A., Beginnende multiple Sklerose und akute Myelitis. Archiv f. Psych. 19, 667.

4. Ebstein, Deutsches Archiv f. klin. Med. 10.

5. Finkelnburg, Über Myeloencephalitis disseminata und Sclerosis multiplex acuta mit anatom. Befund. Deutsche Zeitschr. f. Nervenheilk. 20.

6. Gerhardt, D., Zur Differentialdiagnose der multiplen Sklerose. Deutsche Zeitschr. f. Nervenheilk. 15.

7. Gubler, zitiert nach Westphal. Archiv f. Psych. 3.

8. Hoffmann, J., Die multiple Sklerose des Zentralnervensystems. Deutsche Zeitschr. f. Nervenheilk. 21.

9. Jacob, A., Münch. med. Wochenschr. 1914, S. 562.

10. - u. Fränkel, Zur Pathologie der multiplen Sklerose mit besonderer Berücksichtigung der akuten Formen. Zeitschr. f. d. ges. Neur. u. Psych. 14.

11. Lenhartz, Beitrag zur Kenntnis der akuten Koordinationsstörungen nach akuten Erkrankungen. Berliner klin. Wochenschr. 1883, Nr. 21/22.

12. Le ube, Über multiple inselförmige Sklerose des Gehirns und Rückenmarks. Deutsches Archiv f. klin. Med. 8.

13. Leyden, E., Zeitschr. f. klin. Med. 1. 1880.

14." - Über akute Ataxie. Zeitschr. f. klin. Med. 18.

15. Maas, Über die Beziehungen der Encephalitis non suppurativa zur multiplen Sklerose. Monatsschr. f. Psych. 18.

16. Marburg, Die sogenannte akute multiple Sklerose. Leipzig-Wien 1906.

17. Müller, E., Die multiple Sklerose des Gehirns und Rückenmarks. Jena 1904 .

18. - Handbuch der inneren Medizin 5.

19. - Über einige weniger bekannte Verlaufsformen der multiplen Sklerose. Zentralbl. f. Neurol. u. Psych. 1905.

20. Nonne, Der heutige Standpunkt der Lehre von der Bedeutung der 4 Reaktionen. Deutsche Zeitschr. f. Nervenheilk. 42.

21. - Multiple Sklerose und Facialislähmung. Deutsche Zeitschr. f. Nervenheilk. 60 .

22. Oppenheim, H., Der Formenreichtum der multiplen Sklerose. Deutsche Zeitsehr. f. Nervenheilk. 52.

23. - Zum Kapitel der Myelitis. Berliner klin. Wochenschr. 1891.

24. - Differentialdiagnose der multiplen Sklerose und Pseudosklerose. Deutsche Zeitschr. f. Nervenheilk. 56.

25. - Lehrbuch der Nervenkrankheiten. Berlin 1913.

26. Reichmann, V., Zur Physiologie und Pathologie des Liquor cerebrospinalis. Deutsche Zeitschr. f. Nervenheilk. 4 2. 
280 H. Baumm : Chronische Encephalony clitis disseminata oder multiple Sklerose?

27. Rönne und Wimmer, Akute disseminierte Sklerose. Deutsche Zeitschr. f. Nervenheilk. 46.

28. Schlesinger, Fall von akuter multipler Sklerose nach Nasern. Deutsehe Zeitschr. f. Nervenheilk. 36.

29. - Wiener klin. Wochenschr. 1911, S. 805.

30. Schüle, Hirn-, Rückenmarksklerose. Deutsches Archiv f. klin. Med. 8.

31. Siemerling und Raecke, Beitrag zur Klinik und Pathologie der multiplen Sklerose mit besonderer Berïcksichtigung ihrer Pathogenese. Archiv. f. Psych. 53. 1914.

32. Westphal, Über eine Affektion des Nervensystems nach Pocken und Typhus. Archiv f. Psych. 3.

33. - Multiple Sklerose und Myelitis. Deutsche med. Wochenschr. 1906. 